\title{
Narrativa
}

\section{I vestiti perduti dell'imperatore. La nuova nudità politica del maschile di fine millennio}

\section{Sandro Bellassai}

\section{(2) OpenEdition}

\section{Journals}

\section{Edizione digitale}

URL: https://journals.openedition.org/narrativa/527

DOI: 10.4000/narrativa.527

ISSN: 2804-1224

\section{Editore}

Presses universitaires de Paris Nanterre

\section{Edizione cartacea}

Data di pubblicazione: 1 décembre 2018

Paginazione: 37-48

ISBN: 978-2-84016-325-1

ISSN: $1166-3243$

Notizia bibliografica digitale

Sandro Bellassai, «l vestiti perduti dell'imperatore. La nuova nudità politica del maschile di fine millennio», Narrativa [Online], 40 | 2018, online dal 01 novembre 2021, consultato il 08 décembre 2021. URL: http://journals.openedition.org/narrativa/527 ; DOI: https://doi.org/10.4000/narrativa.527

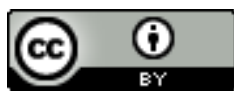

Narrativa est mise à disposition selon les termes de la Licence Creative Commons Attribution 4.0 International. 


\title{
I vestiti perduti dell'imperatore. \\ La nuova nudità politica del maschile di fine millennio
}

\begin{abstract}
RIASSUNTO
L'articolo tratta della rappresentazione mediatica del corpo maschile, nell'Italia a cavallo del millennio, contestualizzandola storicamente in una più generale trasformazione delle relazioni di genere nella contemporaneità. A una nuova "nudità" ora gli uomini, avendo perduto il privilegio dell'invisibilità, non possono evitare di sottostare; si configura così una nuova condizione maschile, nella quale i tradizionali travestimenti retorici della virilità non sono più efficaci, come in passato, a occultare le contraddizioni di un modello identitario sempre più anacronistico, all'indomani della doppia rivoluzione del neocapitalismo e del neofemminismo.
\end{abstract}

\section{RÉSUMÉ}

L'article se concentre sur la représentation médiatique du corps masculin en Italie, au tournant du $\mathrm{XXI}^{\mathrm{e}}$ siècle, en les encadrant dans une transformation plus générale des relations de genre à l'époque contemporaine. Le privilège de l'invisibilité perdu, les hommes ne peuvent plus éviter de se soumettre à une nouvelle "nudité", qui devient ainsi une nouvelle condition masculine. À la suite de la double révolution du néocapitalisme et du néoféminisme, les déguisements rhétoriques traditionnels de la virilité ne permettent plus, comme dans le passé, d'occulter les contradictions d'un modèle identitaire de plus en plus anachronique.

\section{IL DECLINO DELL'INVISIBILITÀ MASCHILE}

Sul piano interpretativo, l'ambito in cui questa riflessione vorrebbe iscriversi è quello delle immagini, degli stereotipi, delle rappresentazioni diffuse della corporeità maschile nell'Italia di fine Novecento; una dimensione, questa, da contestualizzare a sua volta all'interno della più ampia cornice dell'evoluzione storica delle identità, delle relazioni e dei ruoli di genere nel corso degli stessi decenni. Particolare attenzione dedicherò qui ai linguaggi mediatici, che 
assumono proprio fra anni ottanta e novanta una funzione normativa e pedagogica, anche riguardo alla cultura diffusa di genere, molto più incisiva che nel passato.

Da questa prospettiva analitica, la novità più significativa del periodo considerato mi sembra quella di una emersione dall'invisibilità della mascolinità in quanto configurazione identitaria sessuata: negli ultimi due-tre decenni del Novecento, in sintesi, si moltiplicano nella comunicazione pubblica i riferimenti espliciti agli uomini in quanto nomini. L'invisibilità è da sempre privilegio del potere, ovviamente non soltanto in una dimensione di genere; molto spesso, storicamente, un soggetto dominante siè preteso impronunciabile, irriconoscibile nella sua specificità, e quindi anche, in una certa misura, politicamente inattaccabile. È un procedimento che Roland Barthes, a proposito dell'ascesa storica della borghesia come classe dominante, ha chiamato e-nominazione: "la borghesia ha cancellato il suo nome [...] la borghesia si definisce come la classe sociale che non vuole essere nominata".

Nel caso dell'invisibilità maschile (dell'uomo "normale": eterosessuale, bianco, più spesso borghese), è questo un processo da inquadrare entro la più ampia dinamica novecentesca per cui il genere dominante ha visto progressivamente erosa, decennio dopo decennio, la propria potestà tradizionale di affermarsi come soggetto neutro e universale. La maggiore esposizione cui è sottoposto nei linguaggi mediatici il corpo maschile alla fine del millennio, spesso in forme inedite, si configura quindi anche come un indicatore cruciale di questo grande mutamento politico. I tradizionali codici della neutralità, che sin dal tardo Ottocento avevano occultato la parzialità maschile - e dunque anche il corpo sessuato maschile - per porre al riparo la supremazia degli uomini dai venti sovversivi dell'egualitarismo, sembrano ora diventare sempre più inefficaci e anacronistici.

Per buona parte del ventesimo secolo, in estrema sintesi ${ }^{2}$, la partita patriarcale si è giocata anche e soprattutto sul rilancio di un energico virilismo come orizzonte primario delle identità e relazioni di genere: quindi, sull'esaltazione a tutti i livelli della maestà sociale maschile, sulla denigrazione misogina, sulla discriminazione e persecuzione della "devianza" omosessuale. All'interno di tale scenario, la finzione di naturalità e trascendenza della supremazia maschile è

1. Barthes Roland, Miti d'oggi, Torino, Einaudi, 1974 [ed. or. Mythologies, Paris Édition du Seuil, 1970], pp. 218-219. Corsivo nel testo.

2. Rimando, per più ampie considerazioni nel merito, al mio L'invenzione della virilità. Politica e immaginario maschile nell'Italia contemporanea, Roma, Carocci, 2011. 
stato un caposaldo simbolico della sua legittimazione, come - specularmente doveva apparire naturale l'inferiorità delle donne e l'insanità dei "pervertiti". Ma nella seconda parte del secolo l'impalcatura retorica virilista iniziava a crollare sotto i colpi consecutivi della secolarizzazione neocapitalistica, che sin dagli anni sessanta ha identificato l'austerità patriarcale e certe concezioni rigidamente misogine come ostacoli all'espansione dei beni di consumo, e della demolizione neofemminista dell'ordine virilista, al punto che negli anni settanta il virilismo stesso ha toccato il suo minimo storico in termini di legittimazione diffusa.

A partire da questa fase, decade progressivamente anche la funzionalità delle coperture cognitive che avevano più o meno efficacemente occultato la parzialità sessuata maschile, connotando il maschile stesso come un ossimorico genere universale; emerge così una sempre più chiara nudità degli uomini in quanto maschi, ora spogliati dei loro paramenti regali di assolutezza, neutralità, imparzialità. Come nella favola di Andersen, adesso il re è nudo, lo è agli occhi di chiunque e in modo apparentemente irreversibile.

D'altro canto, non sono mancate e non mancano neppure oggi le tenaci insistenze (in verità, non proprio trascurabili) di chi continua ad aggrapparsi saldamente alla vecchia invisibilità maschile; dopo tutto, in effetti, gli uomini rimangono tuttora e sempre il genere del comando. Nella dimensione simbolica legata al mondo militare, ad esempio, che non è certo divenuta residuale di decennio in decennio, si celebra propriamente il livellamento delle individualità all'interno di un corpo collettivo, di una massa in uniforme: e l'uniforme è uno dei veicoli grazie ai quali la realtà concreta di corpi maschili differenti e differentemente vulnerabili si sublima in un'astratta forza collettiva, rivestendosi provvidenzialmente di virilità grazie alla più classica missione guerriera ${ }^{3} . \mathrm{Nel}$ mondo del business e della grande politica internazionale, peraltro, si continua a celebrare una storica standardizzazione in direzione di quello stile corporeo maschile che lo studioso di moda Ted Polhemus, non a caso, definisce come Uomo invisibile:

Dall'età del colonialismo fino ai nostri giorni, per farsi prendere sul serio sulla scena mondiale, un uomo d'affari o un leader politico devono prima rinunciare

3. Aspesi Natalia, "Miles gloriosus. Il ritorno delle uniformi militari nell'immaginario maschile", in MALOSsI Giannino (a cura di), Uomo oggetto. Mitologie, spettacolo e mode della maschilità, Bergamo, Bolis, 2000, pp. 148-153. 
alla propria fisicità e nascondere il corpo dietro l'uniforme, oggi universale, dell'Uomo invisibile: un anonimo completo di cattivo taglio ${ }^{4}$.

Con il collasso tardo-novecentesco del virilismo classico, insomma, il panorama identitario maschile si è fatto straordinariamente frastagliato. Così, nelle culture degli uomini del terzo millennio vecchie strategie, inediti compromessi e nuove rotture più o meno polemiche convivono, si sovrappongono e confliggono; al contempo, in prospettiva storica appare tuttavia evidentissimo il mutamento identitario maschile nel segno di un netto declino - graduale e relativo se vogliamo, ma reale - della tradizionale invisibilità sessuata. Si tratta, senza dubbio, di un mutamento epocale nei rapporti di genere.

È anche necessario considerare, d'altra parte, come tali trasformazioni si iscrivano entro una più generale tendenza culturale alla "corporeizzazione" del sé, secondo certa sociologia, in quanto esasperazione della dimensione fisica nel tentativo di dare stabilità a un'identità individuale sempre più precaria e faticosa: il corpo è ormai il campo di un "progetto", ma anche, e sempre più con la fine del millennio, uno "strumento di contrattazione" sul terreno dell'identità personale. Secondo Federico Boni, in particolare, si tratterebbe di una dinamica culturale complessiva che tende alla "piena auto-regolazione individualizzata del corpo".

\section{EMERSIONI SESSUATE}

Negli ultimi anni del Novecento, in effetti, si registra nella comunicazione di massa (non solo italiana, certamente) una vera e propria affermazione iconografica del corpo maschile, anche nel senso di un'inedita presenza del nudo maschile. Non è certo un caso che una simile nudità maschile emerga dopo il già citato collasso del virilismo classico, e forse pure per tale ragione questa epifania presenta un carattere politico fortemente ambivalente: da un lato costituisce una sorta di abbandono forzato della tradizionale invisibilità, e quindi si configura come una sorta di corto circuito oggettivo dei classici processi virilisti di riproduzione patriarcale; dall'altro, è questo anche un estremo tentativo di riafferma-

4. Polhemus Ted, "L'uomo invisibile", in Malossi Giannino (a cura di), Uomo oggetto, cit., p. 47.

5. Cfr. Giddens Anthony, Modernity and Self-Identity. Self and Society in the Late Modern Age, Cambridge, Polity Press, 1991.

6. Boni Federico, Men's help. Sociologia dei periodici maschili, Roma, Meltemi, 2004, p. 12, anche per la citazione successiva. 
zione di identità di genere nette e polarizzate, a voler scongiurare proprio quella confusione che è da sempre considerata nemica mortale della logica virilista stessa. Quasi che, a fronte di una linea di demarcazione sempre più labile fra generi dicotomicamente intesi, non rimanga altra soluzione che affidarsi sempre più a quello che tipicamente viene considerato il "grado zero" delle identità sessuate, il corpo nudo; nudo, "naturale" e dunque "vero" nel suo essere inconfondibilmente maschile o femminile.

Già nei primi anni novanta, certe novità della rappresentazione mediatica dell'uomo appaiono piuttosto evidenti. Ugo Volli, all'inizio di quel decennio, registra infatti una importante trasformazione quanto alla esponibilità del corpo maschile: il nudo virile inizia ad apparire sistematicamente nell'iconografia pubblicitaria ${ }^{7}$. Anche alla luce di quanto accennato più sopra, un "re nudo" è innanzitutto un sovrano la cui mascolinità sia inevitabilmente visibile, e quindi problematizzabile e discutibile: un simile denudamento è dunque, di fatto, una specie di emersione dalla clandestinità degli uomini in quanto genere. Si tratta probabilmente del massimo grado di vulnerabilità identitaria che il genere maschile abbia raggiunto nella storia recente delle relazioni di genere; e dunque, letteralmente, di una nudità politica a tutto tondo.

Di fatto, l’impossibilità di ricorrere ai tradizionali travestimenti di una neutralità quasi metafisica rende la mascolinità suscettibile - nella propria finitezza politica, nella propria concretezza anche fisiologica - di osservazione e valutazione dall'esterno, conferendo un potere inedito al giudizio delle donne sugli uomini stessi e sui loro corpi. È quindi anche sul piano dell'emersione di uno sguardo desiderante femminile, ora in parte legittimato (ma con mille ambivalenze) nel senso comune diffuso, che si può misurare il mutamento dei rapporti di potere fra i generi negli ultimi decenni del millennio. Si afferma, propriamente, un nuovo regime di visibilità maschile eterosessuale: tendenzialmente, $\mathrm{i}$ corpi degli uomini non possono più evitare di esporsi allo sguardo e al desiderio femminile.

Ma la nuova vulnerabilità maschile (o meglio: la decadenza dei paraventi politici che da lungo tempo la nascondevano), sul finire del Novecento, risente anche di trasformazioni e tensioni che ovviamente non hanno origine solo in una dimensione culturale, o comunque tutta interna alla sfera simbolica del genere in senso stretto. Negli stessi anni, ad esempio, cioè fra il decennio ottanta e novanta, si compie in Occidente la parabola epocale della deindustrializzazione:

7. Cfr. Volli Ugo, "Un corpo violento e artificiale", in ButAzzi Grazietta et alii (a cura di), Virilità e trasgressione, Novara, Istituto Geografico De Agostini, 1992. 
è la fine definitiva del fordismo, e con esso della lunga stagione gloriosa del breadwinner, del capofamiglia maschio come primario o esclusivo procacciatore di reddito. Fenomeni quali la precarietà occupazionale, la stessa disoccupazione ormai cronica, la dequalificazione professionale, tutti massicciamente diffusi a partire da questo periodo, riducono ai minimi termini i margini di riproduzione di quel ruolo sociale che da più di un secolo rappresentava uno dei fondamenti della mascolinità tradizionale.

C'è in particolare un'opera cinematografica che realizza narrativamente, e quasi in presa diretta, una mirabile sintesi di tutto ciò. Prodotta nel 1997, la commedia britannica Full Monty mette in scena prima gli effetti sessuati della fine della città-fabbrica sulle vite concrete di alcuni personaggi maschili, poi la loro angosciata percezione di un mutamento femminile radicale (una mutazione genetica, la definiscono gli attoniti personaggi), infine la decisione economicamente necessitata, per questi ex operai squattrinati, di farsi oggetto dello sguardo desiderante delle donne. Ma soprattutto l'opera di Peter Cattaneo descrive minuziosamente gli effetti della faticosa scelta della nudità sull'autorappresentazione dei protagonisti in quanto uomini, che dunque esperiscono una vera e propria "scoperta" del proprio corpo e della sua vulnerabilità a fronte dello scrutinio delle donne.

Nel film, insomma, uomini "normali" si ritrovano inaspettatamente in una posizione che è stata storicamente esclusiva del genere femminile, quella di doversi sottomettere a una sorta di tribunale dell'altro genere che giudicherà il corpo esaminandolo nei più intimi dettagli (lo strip di Full monty è appunto integrale: l'esposizione dei genitali è l'unica specialità che sentono di poter offrire questi uomini fisicamente non straordinari). Anche sul piano narrativo, in questo caso l'accettazione di un denudamento totale implica quindi a un tempo la rinuncia forzata al privilegio dell'invisibilità di genere (persino in riferimento al proprio stesso sguardo su di sé), il riconoscimento di una potestà giudicante femminile che incide immediatamente sulla propria autostima in quanto maschi, e infine la condivisione fra maschi di un'acuta e dolorosa sensazione di vulnerabilità sessuata.

\section{LA NUDITÀ COME CRISI}

Quanto la nuova visibilità del maschile abbia conseguenze problematiche sul benessere identitario degli uomini stessi, lo testimonia anche la diffusione a livello mediatico di rappresentazioni pessimistiche, sconfortanti, ovvero immediatamente apocalittiche sulla condizione attuale del cosiddetto sesso 
forte. Un elemento molto rilevante di questo scenario complessivo è ad esempio lo sviluppo, sempre negli anni novanta, di un discorso pubblico (ancora una volta, mediatico in particolare) ed esplicito sulla cosiddetta crisi della mascolinità. Si tratta di un tema non proprio inedito - se ne parlava già ampiamente in tutta Europa alla fine dell'Ottocento -, ma che si presenta come un motivo retorico davvero dilagante a partire dagli anni settanta e ottanta del Novecento. È senz'altro interessante notare che, peraltro, il declino dell'invisibilità maschile è stato interpretato come causa ed effetto, a un tempo, di questa percezione diffusa di "crisi". Secondo Marco Alberio,

con l'ingresso del maschio, del corpo maschile e soprattutto della sua sensualizzazione sulla scena mediatica ha preso piede quella che potremmo definire la retorica della crisi maschile [...]. Il concetto della crisi del maschio ha cominciato ad essere un tema ricorrente soprattutto da quando l'uomo e la sua fisicità sono diventati oggetto molto utilizzato nelle rappresentazioni mediatiche. Quindi, è ipotizzabile che questo tema sia fortemente legato alla comunicazione e alla maggiore e nuova visibilità del genere maschile ${ }^{8}$.

Anche per Boni, analogamente,

al discorso sulle "maschilità in crisi" si lega [...] il presunto senso di disagio provocato per le "maschilità egemoni" dall'essere diventate oggetto e non soggetto dell'occhio altrui [...] infatti, la corporeità maschile [...] diviene un problema per le modalità con cui diviene oggetto di consumo ${ }^{9}$.

Dalla fine del millennio siamo entrati ormai chiaramente, aggiunge Boni, in una fase in cui "allo sguardo del 'maschio sulla femmina' si è affiancato quello del 'maschio sul maschio' (oltre a quello della 'femmina sul maschio'), rendendolo socialmente accettabile"10. Compare insomma sulla scena delle identità sessuate, e riceve l'importante legittimazione del Mercato, quello che Tim Edwards studioso britannico del consumo maschile - ha definito "l'uomo allo specchio"11. Il ruolo di garanzia identitaria che svolge in tale ambito la comunicazione pubblicitaria, all'interno di una società che inizia sempre più a delegare al Mercato stesso la ristrutturazione (per non dire la definizione normativa in

8. Alberio Marco, "La metamorfosi del corpo maschile nei media", in Ruspini Elisabetta (a cura di), Uomini e corpi. Una riflessione sui rivestimenti della mascolinità, Milano, Franco Angeli, 2009, p. 215.

9. Boni Federico, Men's help, cit., p. 29. Corsivi nel testo.

10. Ibid., p. 31.

11. Edwards Tim, Men in the Mirror: Men's Fashion, Masculinity, and Consumer Society, London, Bloomsbury, 2016. 
senso stretto) non solo degli assetti politici complessivi, ma anche degli ingredienti simbolici con cui gli individui sono chiamati ad assemblare le proprie identità sessuate in quanto stile, non va in alcun modo sottovalutato. Come ancora osserva Alberio,

si è passati da una fase in cui il corpo maschile (nella sua esposizione) era assente nel testo mediatico ad un'altra in cui la sua presenza è sempre più costante e in aumento. Questo è essenzialmente dovuto al fatto che gli uomini hanno iniziato ad essere percepiti come un importante target di mercato per moltissimi prodotti, non solo quelli a loro tradizionalmente indirizzati: automobili, sigarette, alcolici e rasoi ${ }^{12}$.

Non può dirsi certo nuovo l'utilizzo di una figura maschile come protagonista del messaggio pubblicitario; in qualità di esperto, ad esempio, un uomo compariva spesso come garante del prodotto sin dagli albori della moderna pubblicità televisiva. Ma in quel caso il suo corpo era ben coperto dagli abiti che ne indicavano il ruolo professionale, e che, mentre lo nascondevano allo sguardo, costituivano gli indicatori più importanti dell'autorità virile. Alla fine del Novecento, invece, il mutamento è da questo punto di vista davvero rilevante:

Fino alla metà degli anni Novanta, periodo che coincide con il massimo sviluppo delle TV commerciali, è raro osservare nelle pubblicità italiane e nei programmi televisivi, corpi maschili integralmente o parzialmente esposti. Anche quando si pubblicizzavano prodotti per il corpo e la cura maschile (rasoi, schiume da barba o al massimo profumi) il corpo veniva raramente mostrato nudo. Il più delle volte era coperto da maglietta intima e accappatoio, come mostra ad esempio lo spot Gillette Dopo Barba Contour del 1982 $2^{13}$.

Ma il copione mediatico della "crisi del maschio" non si limita a un generico denudamento: molto più insidiosamente, giunge a investire in modo frontale quello che classicamente è considerato il vero nucleo identitario del vero uomo. Presentato come "testo" autentico dell'identità sessuata degli uomini, il corpo maschile è infatti attraversato mediaticamente, nell'Italia degli anni novanta, anche da tensioni che ne mettono radicalmente in discussione gli attributi più delicati. Ben prima del boom commerciale del Viagra (apparso sul mercato mondiale nel 1998), il tema della "crisi" maschile si àncora dunque alla sfera della sessualità in modo davvero drammatico; la perdita di potenza subìta dalla

12. Alberio Marco, "La metamorfosi del corpo maschile nei media", cit., p. 215.

13. Ibid., p. 220. 
millenaria legittimazione del potere maschile si traduce anche in un'immagine di impotenza fisicamente e impietosamente connotata, fin negli anfratti più imbarazzanti della virilità. Nel nuovo regime della corporeità manifesta, ma anche grazie a una nuova e robusta tendenza cultural-commerciale alla medicalizzazione delle tensioni identitarie socialmente diffuse, un simile senso di non-potere degli uomini, originato in una dimensione più globalmente politica, sfocia insomma anche in una dolorosa diagnosi (letteralmente) clinica destinata a coinvolgere, o addirittura a minacciare, tanto il singolo uomo quanto il genere maschile nel suo complesso ${ }^{14}$.

Così, ad esempio, intorno alla metà degli anni novanta periodici italiani molto diffusi dedicano spazi importanti alle disfunzioni erettili, fenomeno, a quanto si sostiene, in preoccupante aumento: "Sesso debole. Uomini in crisi, un italiano su dieci soffre di impotenza", titola una copertina del Venerdi di Repubblica; "Ciao maschi. Problemi sessuali in aumento", annuncia il supplemento sui temi della salute dello stesso quotidiano; "Provaci ancora uomo. Dossier/Salvare la virilità. Tutto quello che avreste voluto sapere sul sesso ex forte ma non avete mai osato chiedere", recita la titolazione di copertina dell'Espresso. Non si può evitare di registrare come questo avvilente scenario proponga in primo piano, iconograficamente, una molto inquietante controparte sessuata: in alcune di tali immagini infatti compaiono ora un'enorme mano femminile nel gesto di aprire gli slip del povero maschio per scrutarne il contenuto, ora una giovane donna molto divertita mentre punta lo sguardo verso il piccolo pene di un classico Bronzo.

Negli stessi anni, varie altre narrazioni mediatiche investono le intimità maggiormente salienti del corpo maschile, insistendo sul più delicato dei simboli della virilità: in un'analisi della comunicazione pubblicitaria del 1997, ad esempio, si registrava un crescente ricorso non solo a una corporeizzazione, ma precisamente a una genitaližazione del maschile nella pubblicità visiva:

La mascolinità, meglio la virilità, comincia ad essere sottolineata fisicamente fino alla provocazione; la serie di inguini nudi di Benetton piuttosto che la sequenza di membri ben individuabili nelle diverse posizioni, ancorché inguainati in un paio di jeans di "Energie", costituiscono uno stacco rispetto ad una tradizione nella quale la virilità non veniva mai rafforzata attraverso

14. Cfr. Ferrero Camoletto Raffaella, Bertone Chiara, "Introduzione: La medicalizzazione della sessualità maschile: prospettive di analisi e campi di ricerca", in EAD. (a cura di), Le fragilità del sesso forte. Come medicalizzare la maschilità, Milano-Udine, Mimesis, 2016. Per una prospettiva storica più ampia, cfr. inoltre McLAREN Angus, Storia dell'impotenza, Bologna Odoya, 2009 [ed. or. 2007]. 
la dimostrazione genitale, ma lasciata ad una generica prestanza fisica o agli atteggiamenti sociali ${ }^{15}$.

\section{La “Rivoluzione amorale” del Mercato}

Sono tutti esempi, questi, propri di una dimensione della comunicazione direttamente funzionale al consumo. A ben vedere, il nuovo marketing sessuato di fine millennio, mentre trasmette messaggi che sempre più esplicitamente chiamano in causa - per esaltarli, in teoria - i più cari attributi virili, in realtà rappresenta una sconsacrazione tombale della virilità: l'esposizione reiterata del corpo maschile, sottolineando ripetutamente la parzialità sessuata degli uomini, per ciò stesso tende a svestire di assolutezza metafisica la virilità medesima e quindi conduce a un fatidico svelamento della sua vulnerabilità. Da un lato insomma questo vergognoso segreto di Achille finisce per essere, di fatto, sbandierato ai quattro venti; dall'altro, l'espansione di nuovi settori economici legati alla mascolinità come target colloca la stessa identità sessuata degli uomini all'interno diuna vetrina pubblica ormai del tutto incompatibile, tendenzialmente, con lo storico privilegio patriarcale dell'invisibilità di genere.

Se a fine millennio può accadere tutto questo è certamente, come ho già accennato, perché nei decenni precedenti il virilismo come ordine retorico "normale" è stato demolito da una radicale contestazione neofemminista, che ne ha svelato alla radice tutta la natura ideologica: la rivoluzione politica e culturale compiuta dai movimenti delle donne, sin dagli anni sessanta del Novecento, ha infatti definitivamente strappato il genere maschile dal piedistallo di una supremazia indiscussa, strappando al contempo il velo delle gravi contraddizioni intrinseche all'equilibrio identitario tradizionale degli uomini (i quali, senza un saldo ancoraggio alla posizione del comando, accusano immediatamente una sorta di sindrome debilitante della mascolinità: una logica politica destinata fatalmente al tracollo, a fronte dell'affermazione - almeno formale - del principio di uguaglianza).

Ma questo "svelamento" della mascolinità, alla fine del millennio, si compie anche perché il mercato ha finito per individuare nell'invisibilità maschile un limite importante all'espansione sessuata dei beni di consumo; ancora una volta,

15. Caligaris Gianni, "Ostento dunque sono? Il maschio di fine millennio nell'immaginario pubblicitario", "Alfazeta", 63-64 (1997), Derive del maschile. Gli uomini dopo il femminismo, p. 86. 
quindi (come già, lo si è accennato, negli anni sessanta ${ }^{16}$ ), gli interessi "profani”" dell'economia prevalgono impietosamente sulle ultime velleità di sacralità e trascendenza del virilismo. Ha scritto nel 2000 Giannino Malossi:

Il tiro di cui viene fatto oggetto il maschio, sotto forma di obiezioni e messa in discussione della identità di genere e rappresentazione della perplessità maschile contemporanea, è oggi il gioco più frequente nella comunicazione mediatica e nella moda, che di questa è la versione per così dire reificata, trasformata in $\operatorname{merci}^{17}$.

Tuttavia, per fare della mascolinità un vero e proprio target, non è sufficiente che essa venga sconsacrata, secolarizzata, e dunque - nella logica del vecchio statuto patriarcale dell'invisibilità - depotenziata: l'uomo in quanto uomo deve anche, ben più radicalmente, essere messo a fuoco, esibito, interrogato. 亡̀ letteralmente una vivisezione in pubblico, cadenzata sulle esigenze supreme del Mercato; un vero e proprio sacrilegio che non può non configurarsi come l'ultima profanazione della virilità. Siamo qui di fronte, probabilmente (e come altre volte nella storia), a una sorta di rivoluzione amorale: laddove il Mercato, pur perseguendo una strategia moralmente indifferente, cioè essendo tendenzialmente indisponibile a piegarsi a ragioni che le proprie interne finalità economiche non contemplino, produce però di fatto un mutamento politico sui generis, ma reale e profondo, spingendo verso una secolarizzazione oggettiva delle identità e delle relazioni di genere.

Analogamente, le superiori esigenze aziendali non si fanno alcuno scrupolo di contribuire, con la loro potenza mediatica, a un'evoluzione della rappresentazione maschile che concretamente sfocia in una moltiplicazione delle incertezze identitarie degli uomini, se ciò appare funzionale agli interessi produttivi e commerciali prevalenti. Si pensi soltanto, a tale proposito, allo sviluppo impressionante che nuovi consumi legati al corpo, e anche per questo necessariamente sessuati (cosmetici, palestre e centri fitness, integratori alimentari, chirurgia estetica), hanno conosciuto a cavallo del millennio.

Un fenomeno di massa, a ben vedere, che in un angosciante circolo vizioso non può che esporre il genere maschile a un'ulteriore visibilità, davvero inedita per profondità e latitudine sociale. È chiaro, infatti, che tali esperienze corporee

16. Cfr. Bellassai Sandro, "Mascolinità, mutamento, merce. Crisi dell'identità maschile nell'Italia del boom", in CAPuzzo Paolo (a cura di) Genere, generazione e consumi. L'Italia degli anni Sessanta, Roma, Carocci, 2003.

17. Malossi Giannino, "Uomo oggetto", in ID. (a cura di), Uomo oggetto, cit., p. 27. 
si configurano come in se stesse ansiogene, e lo diventano doppiamente nel caso degli uomini: perché spesso sono legate a un senso di inadeguatezza personale e virile, ma anche perché appartengono a una dimensione di cura di sé che è classicamente associata al femminile. L'effetto è allora quello di alimentare sul piano sociale la paurosa sensazione di una confusione crescente tra i generi, e dunque di produrre nuovi timori di svirilizzazione, in un vortice ansiogeno davvero drammatico per l'identità maschile.

Alcune strategie commerciali paiono in effetti guardare precisamente, e piuttosto avidamente, a questo orizzonte di "sessuazione angosciosa". All'inizio del millennio - nel 2000, per l'esattezza - esordisce anche in Italia il fenomeno delle riviste maschili: fa da apripista "Men's Health", edizione italiana di un magazine statunitense, che nella patria del Latin lover vende la bellezza di 400.000 copie al mese $^{18}$. Vari altri periodici analoghi sono apparsi sulla scena successivamente, a comporre un coro comunicativo e pedagogico che si concentra in modo a dir poco maniacale sul corpo, sulla dieta, sulla potenza fisica e sessuale e su semplici ricette per raggiungerla con poca fatica: si tratta in effetti di un vero e proprio Men's help, secondo il titolo del già citato saggio di Boni dedicato a questi nuovi media. Gli esiti a cui giunge una simile narrazione ansiogena possono anche essere paradossali; ecco ad esempio come "Fox Uomo" parla degli inestetismi del petto maschile, e dei modi di risolverli:

La ginecomastia (termine scientifico che indica l'eccessivo ingrandimento delle mammelle nell'uomo) può causare insicurezza e difficoltà nei rapporti con le persone, soprattutto con le donne. Il problema del "seno" non è tipico solo degli uomini in sovrappeso o di mezza età, ma riguarda anche i trentenni attenti all’alimentazione e con una vita dinamica. E quando né la dieta né la ginnastica risolvono il problema, se vuoi proprio esibire un torace asciutto e tonico al punto giusto, puoi prendere in considerazione l'intervento chirurgico ${ }^{19}$.

Il titolo di questo articolo è straordinariamente interessante, ai fini del nostro discorso: "Re di coppe? No, grazie". La sequenza della storica detronizzazione maschile si fa qui davvero micidiale. Da re, a re nudo, a re di coppe: comprensibilmente, è davvero troppo per gli ultimi orfani della famosa virilità italica.

Sandro BeLLASSAI Università di Bologna

18. Cfr. Boni Federico, Men's help, cit., p. 41.

19. Re di coppe? No, grazie, "Fox Uomo", maggio 2003, p. 61. 